Chronic Obstructive Pulmonary Diseases: Journal of the COPD Foundation

\author{
Original Research
}

\title{
St George's Respiratory Questionnaire Score Predicts Outcomes in Patients with COPD: Analysis of Individual Patient Data in the COPD Biomarkers Qualification Consortium Database
}

Hana Müllerova, $\mathrm{PhD}^{1}$ Heather Gelhorn, $\mathrm{PhD}^{2}$ Hilary Wilson, $\mathrm{PhD}^{2}$ Victoria S. Benson, $\mathrm{PhD}^{1}$

Niklas Karlsson, $\mathrm{PhD}^{3}$ Shailendra Menjoge, $\mathrm{PhD}^{4}$ Stephen I. Rennard, $\mathrm{PhD}^{5,6}$ Maggie Tabberer, $\mathrm{MSc}^{1}$

Ruth Tal-Singer, $\mathrm{PhD}^{7}$ Debora Merrill, $\mathrm{MBA}^{8}$ Paul W. Jones, PhD, FRCP, FERS ${ }^{1,9}$

\section{Abstract}

Background: We aimed to estimate the usefulness of a disease specific health status measure, the St George's Respiratory Questionnaire (SGRQ), to predict outcomes in patients with chronic obstructive pulmonary disease (COPD).

Methods: Individual patient-data of 12043 patients from long-term randomized clinical trials (2-4 years' duration) in the COPD Biomarkers Qualification Consortium database were analyzed. The adverse COPD outcomes were: exacerbations of COPD, hospital admissions due to exacerbation and all-cause mortality. Cox proportional hazards regression was used to calculate adjusted hazard ratios (HR) and 95\% confidence intervals (CIs) for quartiles of SGRQ scores at baseline and time to first event, and time from first to second event, where appropriate.

Results: The risk of adverse COPD outcomes increased with each increasing quartile of SGRQ score for all time to first event analyses. When comparing the lowest versus the highest quartile, the event risk (HRs [95\% CIs]) increased by 40\% for exacerbations (1.40 [1.29, 1.51]); 2-fold for hospital admissions (2.01 [1.78, 2.28]) and more than 2 -fold for all-cause mortality $(2.30[1.91,2.78])$. For second event analyses in a subset of eligible patients, these trends persisted albeit with reduced risk estimates for exacerbations.

Conclusions: Among patients with COPD, health status measured by a SGRQ score predicted exacerbations of COPD, hospital admissions due to exacerbations and their recurrence and death after adjustment. These data support the rationale for a health status measure use as a drug development tool and suggest that a health status measure may also have a role in risk assessment for COPD patients in routine medical care.

\begin{abstract}
Abbreviations: St George's Respiratory Questionnaire, SGRQ; chronic obstructive pulmonary disease, COPD; hazard ratios, HRs; confidence interval, CI; COPD Biomarkers Qualification Consortium, CBQC; randomized controlled trials, RCTs; body mass index, BMI; World Health Organization, WHO; forced expiratory volume in 1 second, FEV1; standard deviation, SD; COPD Assessment Test, CAT

Funding Support: Funding for this COPD Biomarkers Qualification Consortium working group was provided by AstraZeneca, BoehringerIngelheim, GlaxoSmithKline, Novartis and Pfizer.

Date of Acceptance: February 1, 2017

Citation: Müllerova H, Gelhorn H, Wilson H, et al. St George's Respiratory Questionnaire score predicts outcomes in patients with COPD: Analysis of individual patient data in the COPD Biomarkers Qualification Consortium Database. Chronic Obstr Pulm Dis. 2017;4(2):141149. doi: https://doi.org/10.15326/jcopdf.4.2.2017.0131
\end{abstract}

1 Research and Development, GlaxoSmithKline, Uxbridge, United Kingdom

2 Evidera, Bethesda, Maryland
3 Research and Development, AstraZeneca, Mölndal, Sweden

4 Boehringer-Ingelheim Pharmaceuticals, Inc., Ridgefield, Connecticut 
5 Division of Pulmonary, Critical Care, Sleep and Allergy, Nebraska Medical Center, Omaha

6 AstraZeneca, Cambridge, United Kingdom

7 Respiratory Therapy Area Unit, Research and Development, GlaxoSmithKline, King of Prussia, Pennsylvania

8 COPD Foundation, Washington, D.C.

9 Institute for Infection and Immunity, St George's University of London, United Kingdom

\section{Address correspondence to:}

Hana Müllerová, PhD

Respiratory Epidemiology

Research and Development

GlaxoSmithKline

Stockley Park West

1-3 Ironbridge Road

Uxbridge, Middlesex, UB11 1BT

United Kingdom

Email: hana.x.muellerova@gsk.com

\section{Keywords:}

St George's Respiratory Questionnaire; SGRQ; COPD outcomes; predictive validity; COPD Biomarkers Qualification Consortium; $\mathrm{CBQC}$

\section{Introduction}

Chronic obstructive pulmonary disease (COPD) is the third leading cause of death in the United States and worldwide. ${ }^{1}$ COPD is characterized by persistent airflow limitation, which is usually of a progressive nature. $^{2}$ In many patients, the course of COPD is punctuated by exacerbations. ${ }^{3}$ Exacerbations of COPD can result in hospital admissions, which are associated with both high clinical impact on patients and substantial economic burden to health care systems. ${ }^{4-6}$ Measures aiming to improve quality of care and to reduce hospitalizations have been introduced in many health care systems. In the United States, targets to reduce readmission before 30 days have been introduced as a way to improve care and reduce direct health care cost. ${ }^{7}$ Identifying patients at high risk of exacerbations of COPD or death is therefore of importance for both patients and health care providers and administrators.

Patient-reported health status is both an important marker of a patient's well-being and a predictive marker for other outcomes, including mortality, hospitalizations and rehospitalizations across a spectrum of diseases. ${ }^{8-11}$ Among patients with COPD, poorer health status was associated with an increased risk of adverse COPD outcomes including exacerbations of COPD and mortality, across a variety of patient populations and settings, including clinical trials and observational studies. $3,12,13$

In this analysis, our objective was to expand this body of literature by quantifying the relationship between baseline health status measured using the St George's Respiratory Questionnaire (SGRQ), and COPD outcomes including exacerbations of COPD, hospital admission due to exacerbation, recurrent exacerbation, recurrent hospital admission due to exacerbation and all-cause mortality. This was accomplished using a large population of patients with COPD identified from an integrated database of individual patient data ( $\mathrm{n}=12043)$ from randomized clinical trials between 2 and 4 years in duration that were pooled in the COPD Biomarkers Qualification Consortium (CBQC) database. The CBQC was specifically formed to support the qualification of novel and widely recognized endpoints as drug development tools in COPD studies.

\section{Methods}

\section{Design and Population}

Using a pre-specified analysis plan, we conducted a longitudinal analysis using prospectively collected individual patient data from 3 long-term (2-4 years) randomized controlled trials (RCTs) that had been integrated into the $C B Q C$ database for SGRQ regulatory qualification. Further details on these clinical trials are available in the online data supplement Table S1. The overall objectives and design of the CBQC initiative, a public-private partnership are described elsewhere. ${ }^{14}$

\section{Health Status Measure: St George's Respiratory Questionnaire}

The SGRQ was chosen, since it is a well-established health status measure used in many interventional and observational studies. The minimal important clinical difference is estimated as a 4 unit difference or change in the SGRQ total score. ${ }^{15}$

The baseline SGRQ scores, at study entry, were stratified into quartiles in order to improve interpretation from a clinical perspective and minimize the effect of any potential non-linear relationship between SGRQ score and the outcomes of interest. To represent the SGRQ score distribution in a broad clinical population of patients diagnosed with COPD, the quartiles were 
determined using baseline SGRQ scores calculated using all the patients in the medium-term studies in the integrated database, comprising of long- term RCTs and observational studies (total $N=19543$ ). Quartiles of SGRQ scores were derived as: 1 st: $<32$, 2 nd: $\geq 32$ and $<46$, 3rd $\geq 46$ and $<60$, 4th $\geq 60$, with the first quartile representing the best health status.

\section{Other Measures}

Details on age, sex, body mass index (BMI) category, World Health Organization (WHO) income category, ${ }^{16}$ smoking status (current or past), duration of COPD, treatment group (summarized as long-acting bronchodilators, inhaled corticosteroid-containing medications, and placebo); and the grade of airflow limitation based on the value of forced expiratory volume $\left(\mathrm{FEV}_{1}\right)$ in 1 second $^{2}$ were also available. In addition, the year of study initiation was included to account for trends in changes in management of COPD over time.

\section{Outcomes}

The following outcomes of COPD were evaluated: (a) exacerbations of COPD, (b) hospital admissions due to COPD, and (c) all-cause mortality. For exacerbations and hospital admissions, both first and second events, were considered. Exacerbations of COPD were defined based on health care utilization, per specific study protocols. Events resulting in a COPD patient being managed with a prescription for an oral corticosteroid and/or antibiotic were classified as moderate exacerbations. Hospital admissions due to exacerbations of COPD, labeled as severe events, required an admission to a hospital with at least 1 overnight stay.

\section{Statistical Analysis}

This is a post-hoc re-analysis of existing clinical trials data. The data analysis plan was approved by the CBQC and all participating pharmaceutical companies. No further scientific or ethics review and approval was required. Data from all patients were analyzed independent of their original allocation to an intervention with an individual medication; however, treatment group (e.g., bronchodilator), was included as a covariate in the analysis.

Summary statistics for each outcome, including event count, rate per patient-year in the study and time to event in days during follow-up, were calculated. Time to event analysis was described using Kaplan-Meier curves. Cox proportional hazards models were employed to estimate hazard ratios (HRs) and 95\% confidence intervals (CIs) for the associations between baseline SGRQ scores (quartiles) and the risk of experiencing an outcome. All analyses were adjusted for age, sex, year of study start with reference group of pre-2002 trials, BMI, WHO income group, treatment group, COPD duration, airflow limitation grade, and smoking status. A test for HRs across quartiles using the mid-point for each SGRQ quartile and assuming a linear trend was applied.

For analysis of each outcome a specific cohort of patients was derived, where patients were excluded from the analysis if they had (1) maximum duration of inclusion in a study $\leq 76$ days; (2) an outcome that occurred $\leq$ day 76; or, (3) missing information for any of the adjustment variables. This decision was made based on the observation that there were many missing outcomes for patients who had dropped out of the study soon after the baseline visit and prior to the common time point for study visit 1 . The reasons for drop-out and the outcomes for these patients were unknown. To minimize information bias caused by these missing data, records of exacerbation events, or hospitalizations occurring prior to day 77 for those participants that had a maximum study day more than 76 days, were also removed.

Analyses were conducted using SAS 9.4 software.

\section{Results \\ Patient Disposition and Selected Baseline Characteristics}

Data from 12043 patients with COPD were integrated (Figure 1); their mean age was 64.7 years (standard deviation [SD] 8.4), 76\% were male and on average they had severe airflow limitation (mean $\mathrm{FEV}_{1}$ predicted of $45.0 \%$, SD 12.7\%). During the 12-month period prior to baseline, $61.3 \%$ experienced at least 1 exacerbation and $18.1 \%$ were admitted to the hospital for COPD. The number of patients with data available for analysis per each outcome was: exacerbation population, $\mathrm{N}=8997$; hospital admission due to exacerbation population, $\mathrm{N}=10562$; all-cause death population, $\mathrm{N}=10850$ (Figure 1).

\section{Distribution of Outcomes of Exacerbations and Mortality During Follow-Up by Baseline SGRQ Score Quartiles}

Overall, the number of exacerbations per patient and mean rate per patient-year in study increased with 


\section{Figure 1. Study Population Flow Diagram}

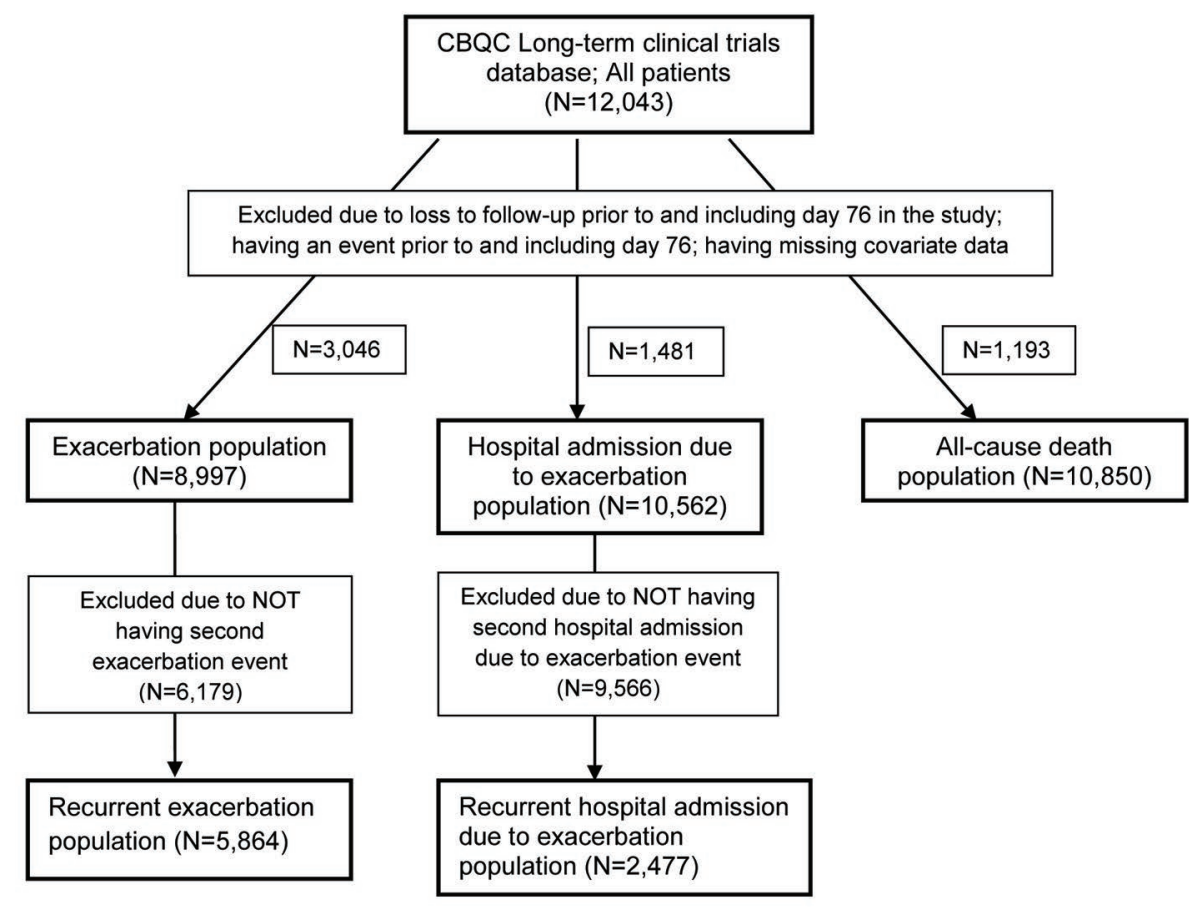

number of events increased from 0.11 to 0.29 events per patient per year with increasing SGRQ quartile. Among those with at least 1 event, the time to first hospital admission was shorter with worse SGRQ score; the difference in median number of days between the first and fourth quartiles was 89 days (532 days versus 443 days); survival time to first hospital admission is described in Figure S2 in the online data supplement. Among patients with more than 1 hospital admission ( $N=2477$ ), the time from first to second also shortened with increasing SGRQ score, with a difference in median number of days between the first and fourth quartiles of 94 days (270 days versus 176 days).

Overall, $10.2 \%$ of 10850 patients died during the follow-up period. Mortality increased with increasing SGRQ quartile, from $6.8 \%$ in the 1 st quartile

worsening baseline SGRQ score quartile (Table 1). The number of patients who reported an exacerbation increased from 57\% (1st quartile: representing best health status) to $70 \%$ (4th quartile), and the mean number of exacerbations increased from 0.60 to 1.03 events per patient per year. Among those with at least 1 exacerbation, the overall median time to first event was 321 days which decreased with worsening SGRQ quartile; the difference between the first and fourth quartiles of SGRQ was 76 days (361 days versus 285 days); survival time to first exacerbation is described in Figure $\mathrm{S} 1$ in the online data supplement. Among patients with more than 1 exacerbation $(\mathrm{N}=5864)$, the median time from first to second event was also shorter with worse SGRQ score; the difference between the first and fourth quartiles was 57 days (211 days versus. 154 days).

Data were available for 10562 patients for the analysis of hospital admissions. This sample was larger than the exacerbation sample because exacerbations occurred more frequently in the $\leq$ day 76 period, so more cases were excluded from the exacerbation analysis. Like the exacerbation population, the number of patients who reported a hospitalization increased from 16\% (1st quartile: representing best health status) to $31 \%$ (4th quartile: worst health status) (Table 2). The mean to $14.3 \%$ in the 4 th quartile; survival time decreased with increasing quartile of SGRQ score (Table 2, Figure S3 in the online data supplement).

\section{Risk of COPD Outcomes by Quartile of Baseline SGRQ Score}

In a multivariable analysis, a significant association between worse health status and increased risk of exacerbation was observed with each successively higher quartile of SGRQ score (trend analysis of HR estimates across quartiles, $p<0.0001$ ) (Table 3 ). The corresponding risk estimates versus the 1st SGRQ quartile of an exacerbation (as HRs [95\% CIs]) were 1.19 $[1.11,1.28]$ for 2 nd quartile, 1.28 [1.19, 1.38] for 3rd quartile, and $1.40[1.29,1.51]$ for 4 th quartile. The risk of a second event also increased with each successive increase in quartile of SGRQ score. When compared to the first SGRQ quartile, HRs [95\% CIs] were 1.18 [1.081.30], 1.20 [1.09-1.32], and 1.26 [1.14-1.40] for 2nd, 3rd, and 4th SGRQ quartiles, respectively; trend analysis HR estimates across SGRQ quartiles, $p<0.0001$.

Similarly, the risk of hospital admission due to an exacerbation increased with worsening health status (Table 3). Each successively higher quartile of SGRQ score conferred an increased risk of first hospitalization (HRs [95\% CIs]) versus 1st quartile: 1.27 [1.11, 1.44] 


\section{Table 1. Distribution of Exacerbations During Follow-up Split by Quartiles of Baseline St George's Respiratory Questionnaire Score}

\begin{tabular}{|c|c|c|c|c|}
\hline & \multicolumn{4}{|c|}{ Baseline SGRQ Score Quartiles } \\
\hline & $\begin{array}{c}1 \text { st } \\
\text { Quartile: } \\
0<32\end{array}$ & $\begin{array}{c}\text { 2nd } \\
\text { Quartile: } \\
32<46\end{array}$ & $\begin{array}{c}\text { 3rd } \\
\text { Quartile: } \\
46<60\end{array}$ & $\begin{array}{c}\text { 4th } \\
\text { Quartile: } \\
\geq 60\end{array}$ \\
\hline Population for Time to First Exacerbation (N) & 1886 & 2611 & 2423 & 2077 \\
\hline \multicolumn{5}{|l|}{ Distribution of Patients by Number of Events (N, \%) } \\
\hline 0 & $806(42.7 \%)$ & $909(34.8 \%)$ & $790(32.6 \%)$ & $628(30.2 \%)$ \\
\hline 1 & $445(23.6 \%)$ & $590(22.6 \%)$ & $533(22.0 \%)$ & $464(22.3 \%)$ \\
\hline 2 & $259(13.7 \%)$ & $404(15.5 \%)$ & $376(15.5 \%)$ & $303(14.6 \%)$ \\
\hline 3 & $138(7.3 \%)$ & $263(10.1 \%)$ & $237(9.8 \%)$ & $209(10.1 \%)$ \\
\hline$\geq 4$ & $238(12.6 \%)$ & $445(17.0 \%)$ & $487(20.1 \%)$ & $473(22.8 \%)$ \\
\hline Mean (SD) Number of Events per Patient per Year & $0.60(0.86)$ & $0.77(1.02)$ & $0.88(1.08)$ & $1.03(1.29)$ \\
\hline \multicolumn{5}{|l|}{ Time to First Exacerbation (days) ${ }^{\mathrm{a}}$} \\
\hline Mean (SD) & $452(322)$ & $432(313)$ & $405(303)$ & $379(294)$ \\
\hline Median & 361 & 338 & 308 & 285 \\
\hline Population for Time From First to Second Exacerbation (N) & 1080 & 1702 & 1633 & 1449 \\
\hline Patients with $\geq 2$ events (N, \%) & $635(58.8 \%)$ & $1112(65.3 \%)$ & $1100(67.4 \%)$ & $985(68.0 \%)$ \\
\hline \multicolumn{5}{|l|}{ Time from First to Second Exacerbation (days) ${ }^{\mathrm{a}}$} \\
\hline Mean (SD) & $283(251)$ & $264(226)$ & $255(228)$ & 231 (219) \\
\hline Median & 211 & 193 & 178 & 154 \\
\hline
\end{tabular}

${ }^{a}$ Among patients with at least 1 qualifying event

SGRQ=St George's Respiratory Questionnaire; $\mathrm{SD}=$ standard deviation

for 2nd quartile, 1.53 [1.35, 1.74] for 3rd quartile and $2.01[1.78,2.28]$ for 4th quartile; trend analysis for HR estimates across SGRQ quartiles $p<0.0001$. The risk of a second hospital admission also increased with poorer health status; however, to a lesser degree (Table 3).

Risk of all-cause mortality was increased more than twice among patients with the poorest health status compared to those with the best; trend analysis for HR estimates across quartiles $p<0.0001$. The corresponding risk estimates (HRs [95\% CIs]) versus 1st quartile were: 1.35 [1.11, 1.64] for 2nd quartile, 1.71 [1.42, 2.07] for 3rd quartile and $2.30[1.91,2.78]$ for 4 th quartile (Table 3).

\section{Discussion}

In this large database of patients with COPD, SGRQ scores at study entry predicted exacerbations of COPD and their recurrence, hospital admissions due to exacerbations, and all-cause death. This predictive capacity was independent of a comprehensive set of baseline patient demographics and markers of COPD severity, including $\mathrm{FEV}_{1}$ and treatment regimen.
These associations were statistically significant; with each successive increase in the quartile of SGRQ baseline score, the risk of an adverse COPD outcome (exacerbation, hospitalization or death) increased. Patients reporting the poorest health status, i.e., SGRQ score of 60 or more, had a $40 \%$ increased risk of experiencing an exacerbation of COPD and a $26 \%$ increased risk of a recurrent episode compared to those with relatively mild impairment $(S G R Q<32)$. Moreover, the risk for hospital admissions for COPD was doubled in the worst SGRQ quartile (4th quartile) and the risk of recurrent admissions was also increased, by $46 \%$. The group with the poorest health status had an increased risk of death of 2.3-fold relative to patients with the best scores. Even patients in the 2nd and 3rd quartiles of SGRQ scores were at significantly increased risk of adverse events compared to those with the mildest impairment of health status (SGRQ score <32).

Unlike hospitalization and all-cause mortality, the difference in risk of exacerbations between patients in the more severe SGRQ quartiles (2nd-4th quartile) was smaller than the difference between patients in 1 st and 2nd quartiles. The reason for this is not clear, 


\section{Table 2. Distribution of Hospital Admission Due to Exacerbation and All-cause Death Events During Follow-up Split by Quartiles of Baseline St George's Respiratory Questionnaire Score}

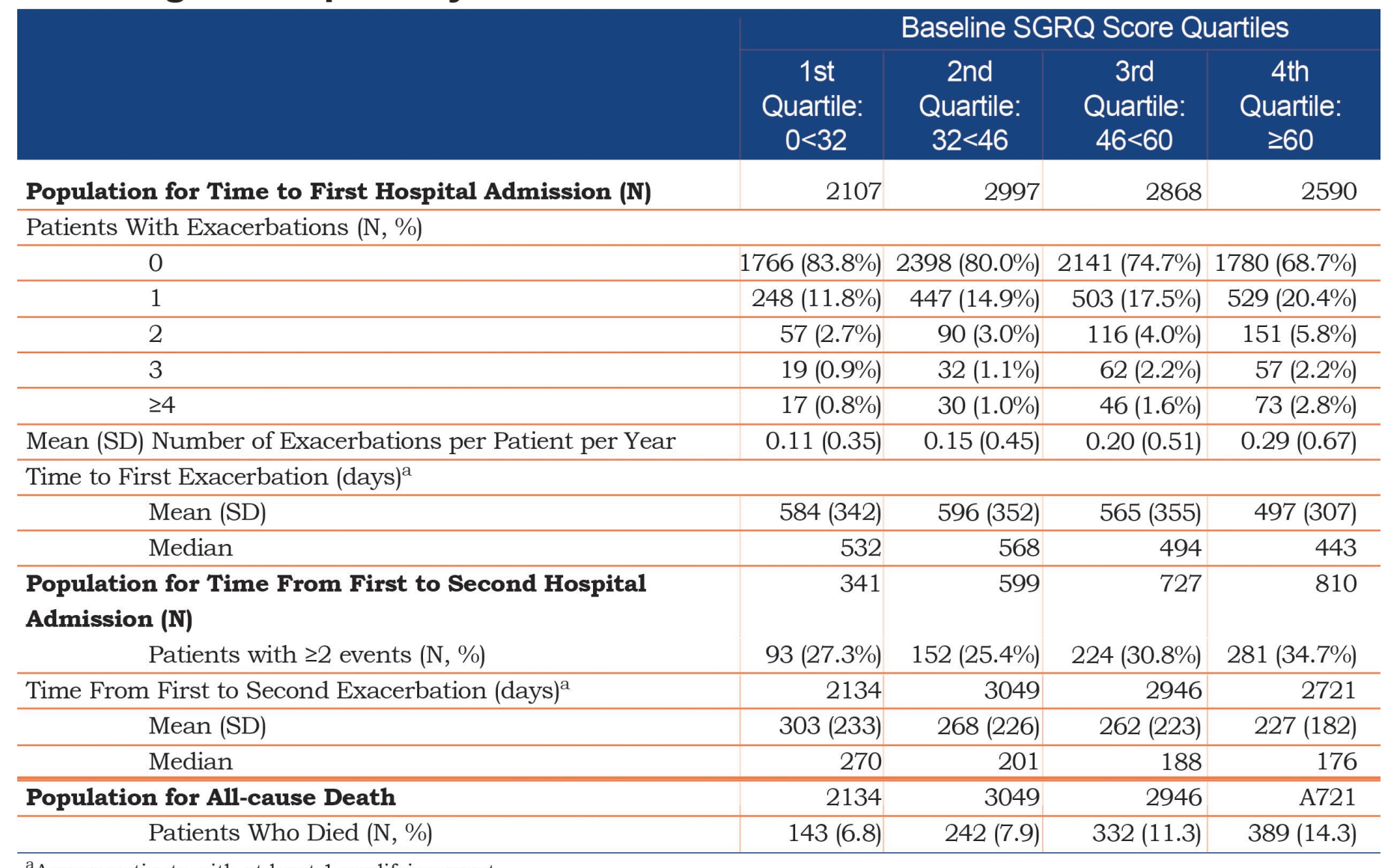

${ }^{a}$ Among patients with at least 1 qualifying event

SGRQ=St George's Respiratory Questionnaire; $\mathrm{SD}=$ standard deviation

\section{Table 3. Risk of COPD Outcomes by Quartile of Baseline St George's Respiratory Questionnaire Score}

\begin{tabular}{|c|c|c|c|c|c|}
\hline $\begin{array}{l}\text { SGRQ } \\
\text { Quartile }\end{array}$ & Exacerbation & $\begin{array}{c}\text { Second } \\
\text { Exacerbation }\end{array}$ & $\begin{array}{l}\text { Hospital } \\
\text { Admission }\end{array}$ & $\begin{array}{l}\text { Second } \\
\text { Hospital } \\
\text { Admission }\end{array}$ & $\begin{array}{c}\text { All-cause } \\
\text { Death }\end{array}$ \\
\hline & $H R(95 \% C I)^{a}$ & $\operatorname{HR}(95 \% C I)^{a}$ & $H R(95 \% C I)^{a}$ & $\operatorname{HR}(95 \% C I)^{a}$ & $H R(95 \% \text { CI })^{a}$ \\
\hline First & 1.00 & 1.00 & 1.00 & 1.00 & 1.00 \\
\hline Second & $\mathbf{1 . 1 9}(1.11-1.28)$ & $\mathbf{1 . 1 8}(1.08-1.30)$ & $\mathbf{1 . 2 7}(1.11-1.44)$ & $\mathbf{1 . 1 0}(0.87-1.40)$ & $\mathbf{1 . 3 5}(1.11-1.64)$ \\
\hline Third & $\mathbf{1 . 2 8}(1.19-1.38)$ & $1.20(1.09-1.32)$ & $1.53(1.35-1.74)$ & $1.18(0.94-1.50)$ & $\mathbf{1 . 7 1}(1.42-2.07)$ \\
\hline Fourth & $1.40(1.29-1.51)$ & $\mathbf{1 . 2 6}(1.14-1.40)$ & $2.01(1.78-2.28)$ & $1.46(1.16-1.83)$ & $\mathbf{2 . 3 0}(1.91-2.78)$ \\
\hline
\end{tabular}

${ }^{a}$ Multivariable Cox proportional hazards analysis producing adjusted HRs and 95\% CIs adjusted for treatment group, year study began, sex, age, BMI, WHO income group, smoking status, COPD duration, and airflow limitation grade.

SGRQ=St George's Respiratory Questionnaire; HR=hazard ratios; CI=confidence intervals; $B M I=$ body mass index;

WHO=World Health Organization 
but it suggests a floor effect in the capacity of the SGRQ to predict exacerbations with any score above the mildest degree of health status impairment. SGRQ scores predicted both recurrent exacerbations and hospitalization, but to a lesser degree compared with the risk of any first event. Several factors may drive this observation. First, we excluded patients who left the study or who experienced 1 of the study outcomes prior to Day 76, which was the common time of the first post baseline study visit within the CBQC SGRQ database. This means that only stable patients were included in the analysis. Second, patients often die or are withdrawn from studies as the result of their exacerbations and hence a survivor bias may have contributed to the increase, although to a lower degree of risk of second, compared to first events. Finally, the time for which individuals were at risk for first events was greater than that of second events.

This study is the first large-scale analysis of the predictive validity of the SGRQ in COPD. It combined data from individual patients participating in 3 large long-term trials and the size of the analysis population is unprecedented in COPD. The use of data obtained in RCTs allowed for a reliable and relatively standardized ascertainment of COPD outcomes through similar definitions of events used in the primary trials. The success of this large-scale analysis is the result of a unique public-private partnership coordinated by the COPD Foundation (a non-profit organization representing patients with COPD) that allowed integration of clinical trial datasets across several pharmaceutical industry-sponsors working in collaboration with academics that provides a model for future data integration efforts. ${ }^{14}$

Several limitations need to be noted in relation to this analysis. It did not include results from the observational studies that were integrated in the CBQC database due to the different outcome definitions and limited longitudinal data availability for some of these studies. Almost $50 \%$ of the clinical trial study populations had missing data concerning pre-baseline exacerbations of COPD, mainly due to differences in the way these data were collected across the trials. This prevented us from assessing the effect of prior exacerbations (i.e., before entry into the trials) on the association between health status and COPD outcomes. Since it is known that previous exacerbation history is predictive of future exacerbations, this is an important limitation. ${ }^{3}$ Further, limited information was available on comorbidities of COPD and, hence, these were also not included in the model. RCTs often recruit selective samples of the clinical population, which may limit the generalizability of these observations. To partly mitigate this bias, the quartiles of SGRQ baseline score were based on both long-term RCTs and observational studies of similar duration. The distribution of SGRQ score in this study population corresponds closely to that observed in a cross-sectional study of 1817 patients with COPD recruited from 5 European countries (median SGRQ score 44 , interquartile range $30-59) .{ }^{17}$

The findings of the current study suggest that health status measurement provides additional prognostic information independent of factors such as age, sex, smoking status, BMI, income group, duration of COPD and airflow limitation and may also be of value in clinical practice. However, since the SGRQ was developed for specialist and research use, it is likely to be too time consuming for routine use in primary care. Newer shorter instruments such as the COPD Assessment Test (CAT) have been developed to overcome this limitation on use of SGRQ. ${ }^{18}$ CAT correlates well with SGRQ scores, and there is also evidence that CAT scores can predict exacerbations. It is yet to be determined whether the CAT can predict adverse outcomes in the same way as the SGRQ. ${ }^{19}$

\section{Conclusions}

Among patients with COPD, a baseline SGRQ score is a significant predictor of exacerbations, hospital admissions and death. The data support the rationale for a health status measure use as a drug development tool and suggest that a health status measure should be a part of risk assessment for COPD patients in routine medical care.

\section{Acknowledgments}

The authors would like to thank Debbie Merrill, COPD Foundation, for managing the review process, and the CBQC for their role in aggregating the data. They would also like to thank Tom Martin of Novartis and Katja Rüdell of Pfizer for their review and oversight through the CBQC Steering Committee, and Pfizer for supporting the $\mathrm{CBQC}$.

\section{Declaration of Interest}

HM, VSB, MT, RTS, PWJ, NK, SM and SIR are employees of the pharma companies who funded this analysis. SIR is an employee of the University of Nebraska Medical 
Center. HG and HW participated in this project as employees of Evidera, a company which performs work for hire for multiple pharmaceutical and device companies in outcomes research, and were funded by the CBQC consortium. DM has nothing to declare. 


\section{References}

1. Burney PG, Patel J, Newson R, Minelli C, Naghavi M. Global and regional trends in COPD mortality, 1990-2010. Eur Respir J. 2015;45(5):1239-1247.

doi: https://doi.org/10.1183/09031936.00142414

2. Global Initiative for Chronic Lung Disease (GOLD) Global Strategy for the Diagnosis, Management and Prevention of COPD. GOLD website. http://www.goldcopd.org. Published 2016. Accessed January 2017.

3. Hurst JR, Vestbo J, Anzueto A, et al. Susceptibility to exacerbation in chronic obstructive pulmonary disease. $N$ Engl J Med. 2010;363:1128-1138.

doi: https://doi.org/10.1056/NEJMoa0909883

4. Ford ES, Murphy LB, Khavjou O, Giles WH, Holt JB, Croft JB. Total and state-specific medical and absenteeism costs of COPD among adults aged $>/=18$ years in the United States for 2010 and projections through 2020. Chest. 2015;147(1):31-45. doi: https://doi.org/10.1378/chest.14-0972

5. Mullerova H, Maselli DJ, Locantore N, et al. Hospitalized exacerbations of COPD: risk factors and outcomes in the ECLIPSE cohort. Chest. 2015;147(4):999-1007.

doi: https://doi.org/10.1378/chest.14-0655

6. Perera PN, Armstrong EP, Sherrill DL, Skrepnek GH. Acute exacerbations of COPD in the United States: Inpatient burden and predictors of costs and mortality. COPD. 2012;9(2):131-141. doi: https://doi.org/10.3109/15412555.2011.650239

7. Mannino DM, Thomashow B. Reducing COPD readmissions: Great promise but big problems. Chest. 2015;147(5):1199-1201. doi: https://doi.org/10.1378/chest.15-0380

8. Heidenreich PA, Spertus JA, Jones PG, et al. Health status identifies heart failure outpatients at risk for hospitalization or death. J Am Coll Cardiol. 2006;47(4):752-756.

doi: https://doi.org/10.1016/j.jacc.2005.11.021

9. Rumsfeld JS, MaWhinney S, McCarthy M, Jr. et al. Health-related quality of life as a predictor of mortality following coronary artery bypass graft surgery. Participants of the Department of Veterans Affairs Cooperative Study Group on Processes, Structures, and Outcomes of Care in Cardiac Surgery. JAMA. 1999; 281(14):1298-1303.

doi: https://doi.org/10.1001/jama.281.14.1298

10. Siersma V, Thorsen H, Holstein PE, et al. Health-related quality of life predicts major amputation and death, but not healing, in people with diabetes presenting with foot ulcers: the Eurodiale study. Diabetes Care. 2014;37(3):694-700.

doi: https://doi.org/10.2337/dc13-1212
11. Spertus JA, Jones P, McDonell M, Fan V, Fihn SD. Health status predicts long-term outcome in outpatients with coronary disease. Circulation. 2002;106:43-49. doi: https://doi.org/10.1161/01.CIR.0000020688.24874.90

12. Celli BR, Locantore N, Yates J, et al. Inflammatory biomarkers improve clinical prediction of mortality in chronic obstructive pulmonary disease. Am JRespir Crit CareMed.2012;185(10):10651072. doi: https://doi.org/10.1164/rccm.201110-1792OC

13. Jones PW, Anderson JA, Calverley PM, et al. Health status in the TORCH study of COPD: Treatment efficacy and other determinants of change. Respir Res. 2011;12:71. doi: https://doi.org/10.1186/1465-9921-12-71

14. Casaburi R, Celli B, Crapo J, et al. The COPD Biomarker Qualification Consortium (CBQC). COPD. 2013;10(3):367-377. doi: https://doi.org/10.3109/15412555.2012.752807

15. Jones PW. St. George's Respiratory Questionnaire: MCID. COPD. 2005; 2(1):75-79.

doi: https://doi.org/10.1081/COPD-200050513

16. World Health Organization. Global health risks: Mortality and burden of disease attributable to selected major risks. World Health Organization website. http://www.who.int/healthinfo/ global_burden_disease/GlobalHealthRisks_report_full.pdf Published 2009. Accessed January 2015.

17. Jones PW, Brusselle G, Dal Negro RW, et al. Health-related quality of life in patients by COPD severity within primary care in Europe. Respir Med. 2011;105(1):57-66. doi: https://doi.org/10.1016/j.rmed.2010.09.004

18. Jones PW, Harding G, Berry P, Wiklund, Chen W-H, Kline Leidy N. Development and first validation of the COPD Assessment Test. Eur Respir J. 2009;34(3):648-654. doi: https://doi.org/10.1183/09031936.00102509

19. Lee SD, Huang MS, Kang J,et al. The COPD assessment test (CAT) assists prediction of COPD exacerbations in high-risk patients. Respir Med. 2014;108(4):600-608. doi: https://doi.org/10.1016/j.rmed.2013.12.014 